Francisco Arnaíz de las

Revillas'

Dolores Sousa ${ }^{2}$

Carmen Ardanuy ${ }^{3}$

Carolina García-Vidal ${ }^{4}$

Miguel Montejo ${ }^{5}$

Regino Rodríguez-Álvarez

Juan Pasquau ${ }^{6}$

Emilio Bouza $^{7}$

José A. Oteo ${ }^{8}$

César Balseiro ${ }^{9}$

Cristina Méndez ${ }^{9}$

Nadia Lwoff ${ }^{9}$

Pedro Llinares ${ }^{2,}$

María Carmen Fariñas ${ }^{1, *}$

on behalf of the SOCRATES

Study Group.

\section{Healthcare-associated pneumonia: a prospective study in Spain}

\author{
'Servicio de Enfermedades Infecciosas, Hospital Universitario Marqués de Valdecilla, University of Cantabria, Santander, Spain \\ ${ }^{2}$ Servicio de Enfermedades Infecciosas, Complexo Hospitalario Universitario A Coruña (CHUAC), Coruña, Spain. \\ ${ }^{3}$ Servicio de Microbiologia, Hospital Universitari de Bellvitge. Institut d'Investigació Biomèdica de Bellvitge (IDIBELL), Uni- \\ versity of Barcelona, L'Hospitalet de Llobregat, Barcelona, Spain. \\ ${ }^{4}$ Servicio de Enfermedades Infecciosas, Hospital Clinic-IDIBAPS, Barcelona, Spain. \\ ${ }_{5}^{5}$ Servicio de Enfermedades Infecciosas, Hospital Universitario de Cruces, Bilbao, Vizcaya, Spain. \\ ${ }^{6}$ Hospital Universitario Virgen de las Nieves, Granada, Spain. \\ ${ }^{7}$ Servicio de Microbiología Clínica y Enfermedades Infecciosas, Hospital General Universitario Gregorio Marañón, Madrid. \\ Instituto de Investigación Sanitaria Gregorio Marañón. CIBER de Enfermedades Respiratorias (CIBERES CB06/06/0058), \\ Madrid, Spain. \\ ${ }^{8}$ Departamento de Enfermedades Infecciosas, Hospital San Pedro, Logroño, La Rioja, Spain. \\ ${ }^{9}$ Pfizer S.L.U, Madrid, Spain
}

\section{Article history}

Received: 23 June 2020; Revision Requested: 9 July 2020; Revision Received: 13 July 2020; Accepted: 15 July 2020;

Published: 22 July 2020

\section{ABSTRACT}

Objective. The aim of the study was to describe the epidemiological characteristics and factors related to outcome in Streptococcus pneumoniae and methicillin-resistant Staphylococcus aureus (MRSA) healthcare-associated pneumonia (HCAP).

Patients and method.: A 3-year prospective observational epidemiological case study of HCAP was conducted in seven Spanish hospitals. Microbiological and patient characteristics and outcomes were collected and classified by causative pathogen into 4 categories: "S. pneumoniae", "MRSA", "Others" and "Unknown". Patients were followed up 30 days after discharge.

Results. A total of 258 (84.6\%) patients were enrolled (170 were men [65.9\%]). Mean age was 72.4 years \pm 15 years (95\% Cl [70.54-74.25]). The etiology of pneumonia was identified in 73 cases (28.3\%): S. pneumoniae in 35 patients (13.6\%), MRSA in 8 (3.1\%), and other microorganisms in 30 patients (11.6\%). Significant differences in rates of chronic obstructive pulmonary disease $(p<0.05)$, previous antibiotic treatment ( $p$ $<0.05)$, other chronic respiratory diseases, inhaled corticosteroids $(p<0.01)$, and Iymphoma $(p<0.05)$ were observed among the four groups. Patients with MRSA pneumonia had received more previous antibiotic treatment (87.5\%). Thirty-three (12.8\%) patients died during hospitalisation; death in $27(81.2 \%)$ was related to pneumonia.

Correspondence:

Dr. Maria Carmen Fariñas.

Infectious Diseases Unit, Hospital Universitario Marqués de Valdecilla

Av. Valdecilla, 25, 39008 Santander, Cantabria, Spain

Phone: +34 942202663

E-mail:mcfarinas@humv.es

*Both authors contributed equally to this manuscript
Conclusions. The etiology of HCAP was identified in only one quarter of patients, with $S$. pneumoniae being the most prevalent microorganism. Patients with chronic respiratory diseases more frequently presented HCAP due to MRSA than to $S$. pneumoniae. Death at hospital discharge was related in most cases to pneumonia.

Keywords: Healthcare-associated pneumonia; S. pneumoniae; methicillin resistant- Staphylococcus aureus.

\section{Neumonía asociada a cuidados sanitarios: un estudio prospectivo en España}

\section{RESUMEN}

Objetivo. Describir las características epidemiológicas y factores relacionados con la neumonía asociada a cuidados sanitarios (NACS) causada por Streptococcus pneumoniae y Staphylococcus aureus resistente a meticilina (SARM).

Pacientes y métodos. Estudio epidemiológico observacional prospectivo de casos a 3 años en siete hospitales españoles. Se recogieron las características microbiológicas y de los pacientes y sus resultados y se clasificaron en función del patógeno causante en 4 categorias: "S. pneumoniae", "SARM", "Otros" y "Desconocido". Al alta, se realizó un seguimiento de 30 dias.

Resultados. Se incluyeron $258(84,6 \%)$ pacientes (170 hombres [65.9\%]; edad media 72,4 años \pm 15 años (95\% IC $[70,54-74,25])$. La etiología de la neumonía se identificó en 73 casos (28,3\%): S. pneumoniae en 35 pacientes (13,6\%), SARM en $8(3,1 \%)$ y otros microorganismos en 30 pacientes $(11,6 \%)$. Hubo diferencias significativas en tasas de enfermedad pul- 
monar obstructiva crónica $(p<0,05)$, tratamiento antibiótico previo $(p<0,05)$, otras enfermedades respiratorias crónicas, corticoides inhalados $(p<0,01)$ y linfoma $(p<0,05)$ entre los cuatro grupos. Los pacientes con NACS causada por SARM recibieron tratamiento antibiótico previo en mayor medida $(87,5 \%)$. Treinta y tres $(12,8 \%)$ pacientes murieron durante la hospitalización; en $27(81,2 \%)$ debido a la neumonía.

Conclusiones. Se identificó la etiología de la NACS en solo un cuarto de los pacientes, siendo $S$. pneumoniae el patógeno más frecuente. En los pacientes con enfermedades respiratorias crónicas fue más frecuente la NACS causada por SARM. La muerte tras el alta hospitalaria se relacionó con la neumonía en la mayoría de los casos.

Palabras clave: neumonía asociada a cuidados sanitarios; S. pneumoniae; Staphylococcus aureus resistente a meticilina.

\section{INTRODUCTION}

Pneumonia has traditionally been classified as either community-acquired (CAP) or hospital-acquired pneumonia (HAP), with both entities being characterised by different microbiological profiles [1]. However, over the years, this classification has become less clear, leading in 2005 to the appearance of a new category called healthcare-associated pneumonia (HCAP). HCAP is used to define pneumonia that develops in non-hospitalised patients who are in frequent contact with the healthcare environment and who appear to be at increased risk of infection with multi-drug resistant (MDR) pathogens $[2,3]$ and a poorer outcome than patients with CAP $[2,4-6]$. As a result, a different approach is required when selecting empiric antibiotic therapy in these cases $[2,3,7-10]$. However, it is important to bear in mind that the concept of HCAP has been the subject of debate in recent years, and that several studies have recently questioned our capacity to identify resistant pathogens [11-14].

One of the challenges in the correct management of patients with pneumonia is achieving an accurate microbiological diagnosis. Despite the availability of several tests, the etiologic agent remains unidentified in at least 50\% of CAP cases [ $[15$, 16]. Globally, Streptococcus pneumoniae is the most frequently identified etiologic agent in both CAP and HCAP patients [17, 18], including in Spain [6, 19]. Importantly, methicillin-resistant Staphylococcus aureus (MRSA) has also been identified as an important causative agent in pneumonia, especially HCAP. Although its incidence is variable in America and Europe, recent studies conducted in Italy and Spain have identified MRSA as a causative agent in 7\%-12.3\% of cases $[7,20]$.

Since an accurate diagnosis is not always possible, we conducted a prospective observational epidemiological study in seven hospitals from different locations across Spain to describe the epidemiological characteristics and factors related to outcome in S. pneumoniae and MRSA HCAP.

\section{PATIENTS AND METHODS}

Study design. A multicentre prospective study was con- ducted between October 2013 and August 2016 in seven hospitals in Spain.

The study was performed in accordance with the Declaration of Helsinki, the protocol was reviewed and approved by the Institutional Ethics Committee of Cantabria (Spain) (reference 24/2013) and at all participating hospitals, according to local standards, and informed consent was obtained from each patient. The study was non-interventional, and patients were managed according to the criteria of their treating physicians.

Patients and definitions. Patients $\geq 18$ years of age admitted with HCAP with new infiltrates on a chest $X$-ray and hospitalised at least for $24 \mathrm{~h}$ in any of the participating hospitals were evaluated. Criteria for bloodstream infection described elsewhere were followed for patient classification [9]. Inclusion criteria were: hospitalisation in an acute care facility for $\geq 2$ days within 90 days before the infection; residence in a nursing home or long-term-care facility; intravenous antibiotic therapy, chemotherapy, or wound care, within 30 days before the infection; or regular hospital visits or haemodialysis [3].

In addition, for inclusion in the study, a sample of blood or normally sterile fluid (pleural fluid, cerebrospinal fluid or peritoneal fluid) with or without a corresponding respiratory sample was required (obtained before antibiotic treatment or in the first 24h after starting antibiotic treatment).

Pneumonia was classified into two categories, complicated or non-complicated, depending on clinical presentation. Complicated pneumonia was defined by the presence of lung parenchymal infiltrates and pleural effusions of any size or character on chest $\mathrm{X}$-ray or computed tomography, and body temperature $>38^{\circ} \mathrm{C}[21]$.

Treatment failure was defined as persistence or progression of clinical symptoms/signs of pneumonia after two days of treatment, or progression of chest X-ray anomalies, or the development of new pulmonary or extra-pulmonary clinical symptoms/signs of pneumonia consistent with an active infection.

Microbiological evaluation. Normally sterile fluids (pleural fluid, cerebrospinal fluid or peritoneal fluid), nasopharyngeal swabs and blood were cultured. S. pneumoniae and MRSA isolates were tested for antibiotic susceptibility by microdilution according to Clinical and Laboratory Standards Institute (CLSI) recommendations [22]. Furthermore, all nasopharyngeal swabs were subjected to real time PCR, targeting lytA for the detection of S. pneumoniae [23]. Pneumococcal urinary antigen was detected using the BinaxNOW kit without concentration (Binax Inc., Portland, ME, USA).

Data collection and follow-up. The following patient data were collected: sex, age, place of residence, admission and discharge date if applicable, epidemiological and medical conditions, microbiological study results, history of vaccination, and outcomes. The Charlson Comorbidity Index (CCI) score was calculated to assess comorbidity [24] and CURB-65 and PORT Severity Index (PSI) scores were calculated to stratify patients according to risk of mortality $[25,26]$. 
Follow-up was performed in outpatient clinics or by telephone thirty days after discharge, and included requirement of a new hospitalisation, whether related or not to the HCAP episode, and mortality, whether related or not to pneumonia.

Data were analysed in two different ways. An initial analysis was conducted according to the presence or absence of S. pneumoniae or MRSA, since S. pneumoniae colonisation is considered a pre-requisite for pneumococcal infections [27] and several studies have confirmed that MRSA nasal colonisation is a risk factor for subsequent infection [28]. To maximise results, all samples that were positive for either colonisation or infection were included. A second analysis according to the causative agent identified in each of the participating centres' laboratories was performed, and results were classified into four different categories: "S. pneumoniae", "MRSA", "Others" and "Unknown".

Statistical analysis. The results were analysed using a commercially available statistical software package (SPSS, version 17.0; SPSS Inc., Chicago, Illinois, USA). Data were expressed as mean value \pm standard deviation (SD) or median value and in- terquartile range (IQR). Chi-squared $\left(\mathrm{X}^{2}\right)$ and Fisher exact test or likelihood ratio were used for categorical variables, and the 2-tailed t test or Mann-Whitney test for continuous variables, as appropriate. Statistical significance was set at $\alpha=0.05$.

\section{RESULTS}

Between October 2013 and September 2016, 305 patients in seven Spanish hospitals were considered for inclusion in the study (Figure 1). Of these, 47 (15.4\%) were ineligible because they did not meet any of the inclusion criteria. A total of 258 (84.6\%) subjects were finally enrolled and subsequently analysed; 170 were men (65.9\%). Patient distribution according to hospital of origin was as follows: 74 (23.7\%) from hospital 1; 78 (30.2\%) from hospital 2; 24 (9.3\%) from hospital 3; 18 (7\%) from hospital 4; 36 (14\%) from hospital 5; 19 (7.4\%) from hospital 6, and $9(3.5 \%)$ from hospital 7. Mean age overall was 72.4 years (SD: 15 years) (95\% Cl [70.54-74.25]) and there were no age differences between HCAP patients colonised or infected with $S$. pneumoniae (72.6 years; $S D=11.6$ ) or MRSA (70.6 years; $S D=17.0$ ).

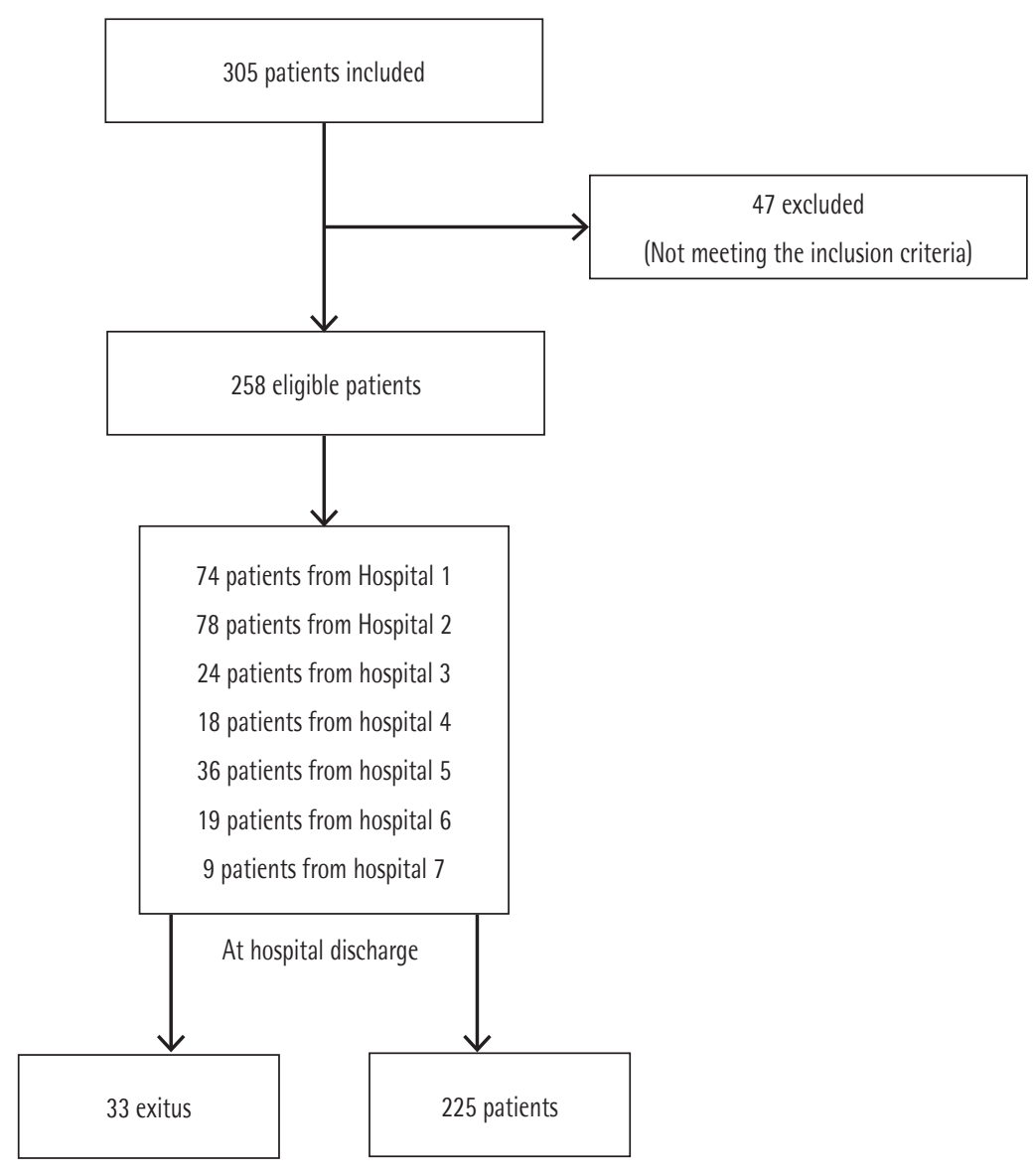

\begin{tabular}{l|l} 
Figura 1 & Flow chart representing study methodology.
\end{tabular} 
Table 1

Patient's clinical characteristics according to the presence or absence of either Streptococcus pneumoniae or methicillin-resistant Staphylococcus aureus.

\begin{tabular}{|c|c|c|c|c|c|c|}
\hline & \multicolumn{2}{|c|}{ S. pneumoniae } & \multirow[t]{3}{*}{$p$-value } & \multicolumn{2}{|c|}{ MRSA } & \multirow[t]{3}{*}{$p$-value } \\
\hline & Presence & Absence & & Presence & Absence & \\
\hline & $n=64$ & $n=194$ & & $n=30$ & $n=228$ & \\
\hline Age, mean \pm SD & $71.22 \pm 13.99$ & $72.78 \pm 15.52$ & 0.218 & $75.3 \pm 15.92$ & $72.01 \pm 15.04$ & 0.276 \\
\hline \multicolumn{7}{|l|}{ Sex, n (\%) } \\
\hline Male & $42(65.6)$ & $128(66)$ & \multirow{2}{*}{0.959} & $20(66.7)$ & $150(65.8)$ & \multirow{2}{*}{0.924} \\
\hline Female & $22(34.4)$ & $66(34)$ & & $10(33.3)$ & $78(34.2)$ & \\
\hline \multicolumn{7}{|l|}{ Relevant history, $\mathrm{n}(\%)$} \\
\hline Hospitalisation (previous 2 months) & $29(45.3)$ & $96(45.9)$ & 0.563 & $16(53.3)$ & $109(47.8)$ & 0.569 \\
\hline Malignancy & $25(39.1)$ & $68(35.1)$ & 0.562 & $5(16.7)$ & $88(38.6)$ & 0.019 \\
\hline COPD & $19(29.7)$ & $60(30.9)$ & 0.852 & $16(53.3)$ & $63(27.6)$ & 0.004 \\
\hline Previous pneumonia & $21(32.8)$ & $55(28.4)$ & 0.497 & $13(43.3)$ & $63(27.6)$ & 0.076 \\
\hline Diabetes & $17(26.6)$ & $54(27.8)$ & 0.843 & $7(23.3)$ & $64(28.1)$ & 0.585 \\
\hline Institutionalised patients & $22(34.4)$ & $48(24.7)$ & 0.133 & $15(50)$ & $55(24.1)$ & 0.003 \\
\hline Heart failure & $13(23.3)$ & $52(26.8)$ & 0.300 & $11(36.7)$ & $54(23.7)$ & 0.124 \\
\hline Previous antibiotic treatment & $38(59.4)$ & $106(54.6)$ & 0.508 & $21(70)$ & $123(53.9)$ & 0.096 \\
\hline Previous chemotherapy (in the last 30 days) & $14(21.9)$ & $32(16.5)$ & 0.330 & $1(3.3)$ & $45(19.7)$ & 0.027 \\
\hline \multirow{2}{*}{\multicolumn{7}{|c|}{$\begin{array}{l}\text { Antibiotic treatment } \\
\text { (7 days prior to hospital admission): }\end{array}$}} \\
\hline & & & & & & \\
\hline Yes & $17(44.7)$ & $52(49.1)$ & \multirow{2}{*}{0.647} & $9(42.9)$ & $60(48.8)$ & \multirow{2}{*}{0.616} \\
\hline No & $21(55.3)$ & $54(50.9)$ & & $12(57.1)$ & $63(51.2)$ & \\
\hline With penicillin & $6(35.3)$ & $9(17.3)$ & 0.119 & $2(22.2)$ & $13(21.7)$ & 0.970 \\
\hline With cephalosporins & $1(5.88)$ & $6(11.5)$ & 0.503 & $3(33.3)$ & $4(6.7)$ & 0.013 \\
\hline With macrolides & $0(0)$ & $5(9.6)$ & 0.184 & $0(0)$ & $5(8.3)$ & 0.369 \\
\hline With quinolones & $5(29.41)$ & $20(38.5)$ & 0.500 & $3(33.3)$ & $22(36.7)$ & 0.846 \\
\hline \multirow{2}{*}{\multicolumn{7}{|c|}{$\begin{array}{l}\text { Antibiotic treatment } \\
\text { (8 days-2 months prior to hospitalisation): }\end{array}$}} \\
\hline & & & & & & \\
\hline Yes & $30(78.9)$ & $86(81.1)$ & \multirow{2}{*}{0.770} & \multirow[t]{2}{*}{$5(23.8)$} & $23(18.7)$ & \multirow{2}{*}{0.584} \\
\hline No & $8(21.1)$ & $20(19.9)$ & & & & \\
\hline With penicillin $+\beta$-lactamase inhibitor & $10(33.3)$ & $19(22.1)$ & 0.221 & $2(12.5)$ & $27(27)$ & 0.214 \\
\hline With cephalosporin & $3(10)$ & $14(16.3)$ & 0.402 & $4(25)$ & $13(13)$ & 0.208 \\
\hline With macrolides & $0(0)$ & $3(3.5)$ & 0.300 & $0(0)$ & $3(3)$ & 0.483 \\
\hline With quinolones & $11(36.7)$ & $25(29.1)$ & 0.439 & $5(31.3)$ & $31(31)$ & 0.984 \\
\hline \multicolumn{7}{|l|}{ Previous pneumococcal vaccinations } \\
\hline Yes & $23(35.9)$ & $80(41.2)$ & \multirow{3}{*}{0.289} & $13(43.3)$ & $90(39.5)$ & \multirow{3}{*}{0.827} \\
\hline No & $25(39.1)$ & $67(34.5)$ & & $11(36.7)$ & $81(35.5)$ & \\
\hline Unknown & $16(25)$ & $47(24.2)$ & & $6(20)$ & $57(25)$ & \\
\hline \multicolumn{7}{|l|}{ Comorbidities, n (\%) } \\
\hline CCl, median (range): & $2(2.56-3.63)$ & $3(2.99-3.65)$ & 0.490 & $3(2.33-3.81)$ & $3(2.99-3.59)$ & 0.603 \\
\hline Absence of comorbidity & $13(20.3)$ & $46(23.7)$ & & $7(23.3)$ & $52(22.8)$ & \\
\hline Low comorbidity & $20(31.3)$ & $41(21.1)$ & 0.255 & $6(20)$ & $55(24.1)$ & 0.875 \\
\hline High comorbidity & $31(48.4)$ & $107(55.2)$ & & $17(56.7)$ & $121(53.1)$ & \\
\hline Heart failure & $3(4.7)$ & $21(10.8)$ & 0.481 & $12(40)$ & $53(23.2)$ & 0.047 \\
\hline Chronic respiratory disease & $27(42.2)$ & $76(39.2)$ & 0.670 & $20(66.7)$ & $83(36.4)$ & 0.001 \\
\hline Moderate/severe chronic liver disease & $2(3.1)$ & $0(0)$ & 0.013 & $0(0)$ & $19(8.3)$ & 0.607 \\
\hline CURB-65 score, $n(\%)$ & & & & & & \\
\hline 0-1 (1.5\% mortality) & $24(39.3)$ & $58(31)$ & & $9(32.1)$ & $73(33.2)$ & \\
\hline $2(9.2 \%$ mortality) & $16(26.2)$ & $65(34.8)$ & 0.370 & $6(21.4)$ & $75(34.1)$ & 0.270 \\
\hline$\geq 3$ (22\% mortality) & $21(34.4)$ & $64(34.2)$ & & $13(46.4)$ & $72(32.7)$ & \\
\hline PSI class, n (\%) & & & & & & \\
\hline I-II & $5(8.8)$ & $16(8.8)$ & & $4(15.3)$ & $17(8)$ & \\
\hline III & $11(19.3)$ & $25(13.8)$ & 0.427 & $4(15.4)$ & $32(15.1)$ & 0.519 \\
\hline IV-V & $41(72)$ & $140(77.3)$ & & $18(69.3)$ & $163(76.9)$ & \\
\hline
\end{tabular}

CCl, Charlson comorbidity index; MRSA, Methicillin-resistant Staphylococcus aureus; PSI, Pneumonia Severity Index; SD, standard deviation; S. pneumoniae, Streptococcus pneumoniae 


\begin{tabular}{|c|c|c|c|c|c|c|c|}
\hline \multirow[t]{4}{*}{ Table 2} & \multicolumn{7}{|c|}{$\begin{array}{l}\text { Hospitalisation-related events and follow-up according to the presence or absence of } \\
\text { either Streptococcus pneumoniae or methicillin-resistant Staphylococcus aureus. }\end{array}$} \\
\hline & & \multicolumn{2}{|c|}{ S. pneumoniae } & \multirow[t]{3}{*}{ p-value } & \multicolumn{2}{|c|}{ MRSA } & \multirow[t]{3}{*}{$p$-value } \\
\hline & & Presence & Absence & & Presence & Absence & \\
\hline & & $n=64$ & $n=194$ & & $n=30$ & $n=228$ & \\
\hline \multicolumn{2}{|c|}{ Treatment failure, $n(\%)$} & $4(6.3)$ & $34(17.5)$ & 0.027 & $6(20)$ & $32(14)$ & 0.386 \\
\hline \multicolumn{2}{|c|}{ Length of stay, mean $\pm S D$} & $9.5 \pm 6.34$ & $11.31 \pm 8.92$ & 0.291 & $11.03 \pm 5.95$ & $10.84 \pm 8.66$ & 0.285 \\
\hline \multicolumn{8}{|c|}{ Antibiogram-guided treatment, $\mathrm{n}(\%)$} \\
\hline Yes & & $25(39.1)$ & $28(14.4)$ & \multirow{2}{*}{0.000} & \multirow[t]{2}{*}{$13(43.3)$} & \multirow[t]{2}{*}{$40(17.5)$} & \multirow{2}{*}{0.001} \\
\hline № & & $39(60.9)$ & $166(85.6)$ & & & & \\
\hline With penic & -lactamase inhibitor & $3(12)$ & $10(35.7)$ & 0.045 & $4(30.8)$ & $9(22.5)$ & 0.547 \\
\hline With cepha & & $10(40)$ & $5(17.9)$ & 0.074 & $2(15.4)$ & $13(32.5)$ & 0.234 \\
\hline With quino & & $1(4)$ & $2(7.1)$ & 0.201 & $0(0)$ & $0(0)$ & 0.398 \\
\hline \multicolumn{8}{|c|}{ Patient status at hospital discharge, $n(\%)$} \\
\hline Cure witho & elae & $54(84.4)$ & $151(77.8)$ & \multirow{3}{*}{0.526} & $20(66.7)$ & $185(81.1)$ & \multirow{3}{*}{0.179} \\
\hline Cure with s & & $4(6.3)$ & $16(8.2)$ & & $4(13.3)$ & $16(7)$ & \\
\hline Death & & $6(9.4)$ & $27(13.9)$ & & $6(20)$ & $27(11.8)$ & \\
\hline \multicolumn{8}{|c|}{ Death related to pneumonia } \\
\hline Yes & & $5(83.3)$ & $22(81.5)$ & \multirow[t]{2}{*}{0.915} & $4(66.7)$ & $23(85.2)$ & \multirow[t]{2}{*}{0.287} \\
\hline No & & $1(16.7)$ & $5(18.5)$ & & $2(33.3)$ & $4(14.8)$ & \\
\hline \multicolumn{8}{|c|}{ Follow-up within $30 \pm 7$ days of discharge, $n(\%)$} \\
\hline Yes & & $49(76.6)$ & $150(77.3)$ & \multirow{2}{*}{0.900} & $19(63.3)$ & $180(78.9)$ & \multirow{2}{*}{0.056} \\
\hline No & & $15(23.4)$ & $44(22.7)$ & & $11(36.7)$ & $48(21.1)$ & \\
\hline \multicolumn{8}{|c|}{ Radiographic resolution } \\
\hline Complete & & $17(35.4)$ & $57(41)$ & \multirow{3}{*}{0.021} & $2(12.5)$ & $72(42.1)$ & \multirow{3}{*}{0.023} \\
\hline Partial & & $7(14.6)$ & $41(29.5)$ & & $4(25)$ & $44(25.7)$ & \\
\hline Unknown & & $24(50)$ & $41(29.5)$ & & $10(62.5)$ & $55(32.2)$ & \\
\hline \multicolumn{8}{|c|}{ Resistant sequelae } \\
\hline Yes & & $47(97.9)$ & $126(90.6)$ & \multirow{3}{*}{0.253} & $14(87.5)$ & $159(93)$ & \multirow{3}{*}{0.648} \\
\hline No & & $1(2.1)$ & $12(8.6)$ & & $2(12.5)$ & $11(6.4)$ & \\
\hline Unknown & & $0(0)$ & $1(0.7)$ & & $0(0)$ & $1(0.6)$ & \\
\hline
\end{tabular}

MRSA, Methicillin-resistant Staphylococcus aureus; SD, standard deviation; S. pneumoniae, Streptococcus pneumoniae

Previous pneumococcal vaccination was reported in 103 of the 258 patients (39.9\%), 93 (90.3\%) of whom had received the polysaccharide vaccine (PPSV), three patients (2.9\%) had received the conjugated vaccine (PCV), one patient (1\%) had received both PCV + PPSV, and the type of vaccine was unknown in six cases (5.8\%).

Patient characteristics according to presence or absence of either S. pneumoniae or MRSA. Of the 258 samples tested by PCR for $S$. pneumoniae identification, 53 were positive. The remaining 205 negative specimens were further tested, yielding one culture-positive sample and 10 BINAX-positive samples. As a result, 64 cases (24.9\%) of S. pneumoniae were identified. Furthermore, the serotype was identified in 14 of these samples; the identified serotypes included ST11A, $n=3,21.4 \%$; ST8, $n=$ $2,14.3 \%$; and one case each of the following: ST1, ST7F, ST6C, ST9N, ST10A, ST16F, ST22, ST31, ST35B ( $n=1,7.1 \%$ for each).

MRSA was identified in 30 patients (11.6\%), six of which were isolated from normally sterile fluid samples such as blood or pleural fluid, or from the corresponding respiratory sample and nasopharyngeal exudates in the remaining 24 .

Clinical data according to the presence or absence of either S. pneumoniae or MRSA are shown in Table 1. As observed among the patients with significant medical histories, malignancy and previous chemotherapy (in the last 30 days) were more frequent in patients in whom MRSA was not isolated, 
Table 3 Patient's clinical characteristics according to causative pathogen

\begin{tabular}{|c|c|c|c|c|c|c|}
\hline & $\begin{array}{l}\text { S. pneumoniae } \\
\qquad(n=35)\end{array}$ & $\begin{array}{l}\text { MRSA } \\
(n=8)\end{array}$ & $\begin{array}{l}\text { Other microorganisms } \\
\qquad(n=30)\end{array}$ & $\begin{array}{l}\text { Unknown } \\
(n=185)\end{array}$ & $\begin{array}{c}\text { Total } \\
(\mathrm{n}=258)\end{array}$ & $p$-value \\
\hline Age, mean $\pm S D$ & $72.57 \pm 11.60$ & $70.63 \pm 13.15$ & $73.93 \pm 17.03$ & $72.19 \pm 15.58$ & $72.40 \pm 15.15$ & 0.780 \\
\hline \multicolumn{7}{|l|}{ Sex, n (\%) } \\
\hline Male & $24(68.6)$ & $7(87.5)$ & $19(63.3)$ & $19(63.3)$ & $170(65.9)$ & \multirow[t]{2}{*}{0.522} \\
\hline Female & $11(31.4)$ & $1(12.5)$ & $11(36.7)$ & $11(36.7)$ & $88(34.1)$ & \\
\hline \multicolumn{7}{|l|}{ Patient-related factors, n (\%) } \\
\hline Hospitalisation (previous 2 months) & $17(48.6)$ & $5(62.5)$ & $19(63.3)$ & $84(45.4)$ & $125(48.4)$ & 0.266 \\
\hline Malignancy & $17(48.6)$ & $4(50)$ & $9(30)$ & $63(34.1)$ & $93(36)$ & 0.290 \\
\hline COPD & $12(34.3)$ & $3(37.5)$ & $16(53.3)$ & $48(25.9)$ & $79(30.6)$ & $<0.05$ \\
\hline Previous pneumonia & $8(22.9)$ & $2(25.0)$ & $10(33.3)$ & $56(30.3)$ & $76(29.5)$ & 0.772 \\
\hline Diabetes & $7(20)$ & $1(12.5)$ & $8(26.7)$ & $55(29.7)$ & $71(27.5)$ & 0.462 \\
\hline Institutionalised patients & $9(25.7)$ & $2(25)$ & $11(36.7)$ & $48(25.9)$ & $70(27.1)$ & 0.686 \\
\hline Heart failure & $7(20)$ & $1(12.5)$ & $10(33.3)$ & $47(25.4)$ & $65(25.2)$ & 0.510 \\
\hline Previous antibiotic treatment & $21(60)$ & $7(87.5)$ & $21(70)$ & $95(51.4)$ & $144(55.8)$ & $<0.05$ \\
\hline \multicolumn{7}{|c|}{ Antibiotic treatment (7 days prior to hospital admission): } \\
\hline Yes & $9(42.9)$ & $2(28.6)$ & $8(38.1)$ & $50(52.6)$ & $69(47.9)$ & 0.396 \\
\hline With penicillins & $3(33.3)$ & $1(50)$ & $0(0)$ & $5(10)$ & $15(21.7)$ & 0.149 \\
\hline With cephalosporins & $0(0)$ & $1(50)$ & $0(0)$ & $6(12)$ & $7(10.1)$ & 0.120 \\
\hline With macrolides & $0(0)$ & $0(0)$ & $1(12.5)$ & $4(8)$ & $5(7.2)$ & 0.579 \\
\hline With quinolones & $2(22.2)$ & $1(50)$ & $5(62.5)$ & $17(34)$ & $25(36.2)$ & 0.340 \\
\hline \multicolumn{7}{|l|}{ Antibiotic treatment } \\
\hline \multicolumn{7}{|l|}{ (8 days-2 months prior to hospitalisation): } \\
\hline Yes & $16(76.2)$ & $6(85.7)$ & $18(85.7)$ & $76(80)$ & $116(80.6)$ & 0.055 \\
\hline With aminopenicillins & $7(43.8)$ & $1(16.7)$ & $3(16.7)$ & $18(23.7)$ & $29(25)$ & 0.296 \\
\hline With cephalosporins & $1(6.3)$ & $1(16.7)$ & $4(22.2)$ & $11(14.5)$ & $17(14.7)$ & 0.745 \\
\hline With macrolides & $0(0)$ & $0(0)$ & $1(5.6)$ & $2(2.6)$ & $3(2.6)$ & 0.467 \\
\hline With quinolones & $6(37.5)$ & $3(50)$ & $7(38.9)$ & $20(26.3)$ & $36(31)$ & 0.267 \\
\hline \multicolumn{7}{|l|}{ Previous pneumococcal vaccinations } \\
\hline Yes & $14(40)$ & $2(25)$ & $12(40)$ & $75(40.5)$ & $103(39.9)$ & \multirow{3}{*}{0.951} \\
\hline No & $13(37.1)$ & $4(50)$ & $12(40)$ & $63(34.1)$ & $92(35.7)$ & \\
\hline Unknown & $8(22.9)$ & $2(25)$ & $6(20)$ & $47(25.4)$ & $63(24.4)$ & \\
\hline \multicolumn{7}{|c|}{ Current inhaled corticosteroid treatment, $\mathrm{n}(\%)$} \\
\hline Yes & $12(34.3)$ & $2(25)$ & $13(43.3)$ & $32(17.3)$ & $59(22.9)$ & \multirow{2}{*}{$<0.01$} \\
\hline No & $23(65.7)$ & $6(75)$ & $17(56.7)$ & $153(82.7)$ & $199(77.1)$ & \\
\hline \multicolumn{7}{|l|}{ Comorbidities, n (\%) } \\
\hline $\mathrm{CCl}$, median (range): & $2(2.44-4.01)$ & $3.5(1.48-5.77)$ & $3(2.91-3.59)$ & $3(2.91-3.59)$ & $3(2.99-3.54)$ & 0.973 \\
\hline Absence of comorbidity & $6(17.1)$ & $2(25)$ & $5(16.7)$ & $46(24.9)$ & $59(22.9)$ & \multirow{3}{*}{0.955} \\
\hline Low comorbidity & $13(37.1)$ & $0(0)$ & $6(20)$ & $42(22.7)$ & $61(23.6)$ & \\
\hline High comorbidity & $16(45.7)$ & $6(75)$ & $19(63.3)$ & $97(52.4)$ & $138(53.5)$ & \\
\hline Chronic respiratory disease & $17(48.6)$ & $4(50)$ & $18(60)$ & $64(34.6)$ & $103(39.9)$ & $<0.05$ \\
\hline Lymphoma & $6(17.1)$ & $0(0)$ & $0(0)$ & $13(7)$ & $19(7.4)$ & $<0.05$ \\
\hline \multicolumn{7}{|l|}{ CURB-65 score, n (\%) } \\
\hline $0-1$ & $14(41.2)$ & $3(50)$ & $9(31)$ & $56(31.3)$ & $82(33.1)$ & \multirow{3}{*}{0.371} \\
\hline 2 & $10(29.4)$ & $3(50)$ & $9(31)$ & $59(33)$ & $81(32.7)$ & \\
\hline$\geq 3$ & $10(29.4)$ & $0(0)$ & $11(37.9)$ & $64(35.8)$ & $85(34.3)$ & \\
\hline \multicolumn{7}{|l|}{ PSI class, n (\%) } \\
\hline$|-| \mid$ & $2(6.3)$ & $0(0)$ & $3(10.3)$ & $16(9.5)$ & $21(8.8)$ & \multirow{3}{*}{0.691} \\
\hline III & $6(18.8)$ & $3(37.5)$ & $3(10.3)$ & $24(4.2)$ & $36(15.1)$ & \\
\hline IV-V & $24(75)$ & $5(62.5)$ & $23(79.3)$ & $129(76.3)$ & $181(76.1)$ & \\
\hline \multicolumn{7}{|c|}{ Presentation of pneumonia at hospital admission } \\
\hline Non-complicated pneumonia & $14(40)$ & $3(37.5)$ & $6(20)$ & $86(46.5)$ & $109(42.2)$ & \multirow{2}{*}{0.043} \\
\hline Complicated pneumonia & $21(60)$ & $5(62.5)$ & $24(80)$ & $99(53.5)$ & $149(57.8)$ & \\
\hline Presentation of pneumonia at hospital a & & & & & & \\
\hline Non-complicated pneumonia & $5(41.7)$ & $1(33.3)$ & $2(12.5)$ & $24(50)$ & $32(40.5)$ & 0043 \\
\hline Complicated pneumonia & $7(58.3)$ & $2(66.7)$ & $14(87.5)$ & $24(50)$ & $47(59.5)$ & \\
\hline
\end{tabular}

CCI, Charlson Comorbidity Index; COPD, Chronic Obstructive Pulmonary Disease; MRSA, Methicillin-resistant Staphylococcus aureus; PSI, Pneumonia Severity Index; S. pneumoniae, Streptococcus pneumoniae; 


\begin{tabular}{|c|c|c|c|c|c|c|c|}
\hline \multirow[t]{2}{*}{ Table 4} & \multicolumn{7}{|c|}{ Hospitalisation-related events and follow-up according to pneumonia-causing pathogen } \\
\hline & & $\begin{array}{l}\text { S. pneumoniae } \\
\qquad(\mathrm{n}=35)\end{array}$ & $\begin{array}{l}\text { MRSA } \\
(n=8)\end{array}$ & $\begin{array}{l}\text { Others microorganisms } \\
\qquad(\mathrm{n}=30)\end{array}$ & $\begin{array}{l}\text { Unknown } \\
(n=185)\end{array}$ & $\begin{array}{c}\text { Total } \\
(n=258)\end{array}$ & p-value \\
\hline Treatment fai & $(\%)$ & $3(8.6)$ & $2(25)$ & $4(13.3)$ & $29(15.7)$ & $38(14.7)$ & 0.576 \\
\hline Length of sta & ), mean $\pm S D$ & $9.57 \pm 6.77$ & $22.13 \pm 16.31$ & $13.17 \pm 10.61$ & $10.24 \pm 7.39$ & $10.86 \pm 8.38$ & 0.005 \\
\hline \multicolumn{8}{|c|}{ Patient status at hospital discharge, $n(\%)$} \\
\hline Cure witho & Ielae & $30(85.7)$ & $3(37.5)$ & $26(86.7)$ & $146(78.9)$ & $205(79.5)$ & \\
\hline Cure with & & $2(5.7)$ & $3(37.5)$ & $3(10)$ & $12(6.5)$ & $20(7.8)$ & 0.047 \\
\hline Death & & $3(8.6)$ & $2(25)$ & $1(3.3)$ & $27(14.6)$ & $33(12.8)$ & \\
\hline \multicolumn{8}{|c|}{ Death related to pneumonia } \\
\hline Yes & & $3(100)$ & $0(0)$ & $1(100)$ & $23(85.2)$ & $27(81.2)$ & 0.034 \\
\hline № & & $0(0)$ & $2(100)$ & $0(0)$ & $4(14.8)$ & $6(18.2)$ & \\
\hline \multicolumn{8}{|c|}{ Follow-up within $30 \pm 7$ days of discharge, $n(\%)$} \\
\hline Yes & & $29(82.9)$ & $4(50)$ & $28(93.3)$ & $138(74.6)$ & $199(77.1)$ & 0.020 \\
\hline No & & $6(17.1)$ & $4(50)$ & $2(6.7)$ & $47(25.4)$ & $59(22.9)$ & \\
\hline \multicolumn{8}{|c|}{ Radiographic resolution } \\
\hline Complete & & $15(51.7)$ & $1(25)$ & $9(36)$ & $49(38)$ & $74(39.6)$ & \\
\hline Partial & & $4(13.8)$ & $1(25)$ & $4(16)$ & $39(30.2)$ & $48(25.7)$ & 0.315 \\
\hline Unknown & & $10(34.5)$ & $2(50)$ & $12(48)$ & $41(31.8)$ & $65(34.8)$ & \\
\hline \multicolumn{8}{|c|}{ Resistant sequelae } \\
\hline Yes & & $1(3.4)$ & $1(25)$ & $3(12)$ & $173(92.5)$ & $13(7)$ & \\
\hline No & & $28(96.6)$ & $3(75)$ & $22(88)$ & $120(93)$ & $48(25.7)$ & 0.731 \\
\hline Unknown & & $10(34.5)$ & $2(50)$ & $12(48)$ & $41(31.8)$ & $65(34.8)$ & \\
\hline
\end{tabular}

MRSA, Methicillin-resistant Staphylococcus aureus; SD, standard deviation; S. pneumoniae, Streptococcus pneumoniae

while institutionalisation and COPD were more common in the MRSA-positive patient group. No differences in any comorbidities were detected according to the presence or absence of $S$. pneumoniae. No differences were observed in previous antibiotic treatment, regardless of the presence or absence of either S. pneumoniae or MRSA. However, treatment with cephalosporins seven days prior to hospital admission was significantly more frequent in patients with MRSA ( $n=3 ; 33.3 \%$ vs. $n=$ $4 ; 6.7 \%, p<0.05)$. Previous pneumococcal vaccination was reported in 23 patients (35.9\%) in the S. pneumoniae group; of these, 22 (95.7\%) had received the polysaccharide vaccine (PPSV). In the MRSA group, 13 patients (43.3\%) had previously received pneumococcal vaccination; of these, 12 (92.3\%) had received PPSV and only one patient had received the conjugated vaccine $(7.7 \%)$.

$\mathrm{CCl}$ score was also calculated, and no differences were found between the groups colonised or infected with either $S$. pneumoniae or MRSA and their respective control groups. Interestingly, specific comorbidities were significantly more frequent in groups that were positive for any of the pathogens, including the incidence of heart failure and chronic respiratory disease in the MRSA-positive group ( $n=12 ; 40 \%$ vs. $n=53$;
$23.2 \%, p<0.05 ; n=20 ; 66.7 \%$ vs. $n=83 ; 36.4 \%, p=0.001$ respectively), and the incidence of moderate/severe chronic liver disease in the $S$. pneumoniae-positive group $(n=2 ; 3.1 \%$ vs. $n=0 ; 0 \%, p<0.05$ ).

No significant differences in CURB-65 and PSI scores were reported between the groups.

Outcomes according to the presence or absence of either S. pneumoniae or MRSA. Relevant data are presented in Table 2. Treatment failure rates were higher in patients not infected or colonised by S. pneumoniae ( $n=34 ; 17.5 \%$ vs. $n=4$; $6.3 \%, p<0.05$ ). There were no differences in the mean length of stay (LOS) among the groups. Treatment was guided by antibiogram significantly more often in groups in which either $S$. pneumoniae or MRSA were identified $(p<0.001$ and $p=0.001$, respectively). Treatment with a penicillin plus a $\beta$-lactamase inhibitor was more common in the group in which $S$. pneumoniae was not identified ( $n=10 ; 35.7 \%$ vs. $n=3 ; 12 \%, p<0.05)$.

Although death was more frequently reported in the absence of either S. pneumoniae ( $n=27 ; 13.9 \%$ vs. $n=6 ; 9.4 \%)$ or MRSA ( $n=27 ; 11.8 \%$ vs. $n=6 ; 20 \%)$, the differences were not statistically significant. 
Patient characteristics according to causative agent. The etiology of pneumonia was identified in 73 of the 258 patients (28.3\%). S. pneumoniae was identified in 35 cases $(13.6 \%)$, MRSA in $8(3.1 \%)$, and other microorganisms were identified in 30 cases $(11.6 \%)$. In the latter group, the most common microorganisms were Escherichia coli $(n=5,16.7 \%)$, followed by Klebsiella pneumoniae $(n=4,13.3 \%)$ and Haemophilus influenzae $(n=3,10 \%)$.

Clinical data are shown in Table 3. Among patient-related factors, the incidence of chronic obstructive pulmonary disease (COPD) $(n=79 ; 30.6 \%)$ differed significantly depending on the HCAP causative agent $(p<0.05)$ and was higher in the group of microorganisms other than S. pneumoniae and MRSA ("Others" group). A total of 144 patients (55.8\%) had received previous antibiotic treatment, with a higher prevalence in the MRSA group $(n=7 ; 87.5 \%)$ compared to the other categories $(p<0.05)$. Regarding comorbidity, there was no difference in the $\mathrm{CCl}$ score among the analysed groups, with more than half of the patients showing high comorbidity $(n=138 ; 53.5 \%)$. However, the incidence of chronic respiratory disease and lymphoma was significantly higher in the "Others" and "S. pneumoniae" groups, respectively ( $p<0.05$ in both cases).

No significant differences among groups were reported in comorbidity measured with the CURB-65 and PSI scores. The most frequent comorbidity was complicated pneumonia $(n=$ $149 ; 57.8 \%)$, that also showed differences among the groups (S. pneumoniae: $n=21 ; 60 \%$; MRSA: $n=5 ; 62.5 \%$; Others: $n=24 ; 80 \%$; Unknown: $n=99$; 53.5\%. $p<0.05$ ). Complicated pneumonia was also more common than non-complicated pneumonia in the 79 patients with COPD $(n=47 ; 59.5 \%$ and $n=32 ; 40.5 \%$ respectively), and the incidence of complicated pneumonia was also significantly related to the etiology of the episode (S. pneumoniae: $n=7 ; 58.3 \%$; MRSA: $n=2 ; 66.7 \%$; Others: $n=14 ; 87.5 \%$; Unknown: $n=24 ; 50 \%$. $p<0.05$ ).

Outcomes according to HCAP causative agent. Relevant data are presented in Table 4. Thirty-eight patients (14.7\%) were treatment failures. Mean LOS was 10.86 days \pm 8.38 days [95\% $\mathrm{Cl}(9.83-11.89)]$ and differed according to the etiology of pneumonia $(p<0.01)$, with the longest stays recorded in the MRSA group [22.13 days \pm 16.31 days; $95 \% \mathrm{Cl}$ (8.49-35.76)] compared to the other categories.

Overall, 33 (12.8\%) patients died during hospitalisation, $27(81.2 \%)$ of which were considered related to pneumonia. Of these cases, 3 were caused by S. pneumoniae, one by other microorganisms, and in 23 cases the etiology was unknown. These differences were statistically significant $(p<0.05)$.

A follow-up visit within $30 \pm 7$ days of discharge was successfully performed in 199 patients (77.1\%).

\section{DISCUSSION}

Our data provides an updated overview of the epidemiological status of HCAP in Spain. To our knowledge, this is the first study in which these patients were evaluated according to the etiology of pneumonia and according to the presence or absence of two of the most relevant pathogens implicated in the disease, S. pneumoniae and MRSA.

Our results show that $S$. pneumoniae is the most frequent causative agent of pneumonia identified in our population, confirming the results of previous studies conducted in Spain $[6,19,29]$, United Kingdom [30] and Japan [4]. However, it is interesting to observe that the etiology of pneumonia could not be determined in most patients, something that underlines two diagnostic-related issues widely discussed in the literature: the lack of sensitivity of conventional cultured-based methods and the difficulty of obtaining good quality specimens from the lower respiratory tract $[31,32]$. Taking into account these problems, we performed an analysis based on the causative agent, and also included patients in whom etiology could not be determined.

In our series, a previous history of COPD was the third most frequent clinical characteristic reported. COPD also showed significant differences when pneumonia was classified according to etiology, being most common in the "Others" group. Interestingly, chronic respiratory diseases identified at presentation was the comorbidity that was also most frequent in patients with pneumonia due to causative agents other than MRSA or S. pneumoniae. Our results confirmed that, on the one hand, COPD is common in HCAP patients. Indeed, previous studies have demonstrated that its incidence as a comorbid condition is comparable to [19] or even higher than in CAP patients [29], in whom COPD can also be considered as the most frequent comorbidity associated with the development of pneumonia [33]. In view of the available data, it is tempting to think that this might also be the case in HCAP patients. On the other hand, although S. pneumoniae is one of the most frequently identified pathogens both in stable periods and in exacerbations in COPD, our results suggest that HCAP patients with this pathology may be exposed to a greater potential risk of presenting colonisation or infections by other microorganisms.

Another important factor was prior use of antibiotics, and indeed, this is one of the criteria that may define an HCAP patient [3]. Interestingly, we found significant differences among the analysed groups, with the MRSA group accounting for a higher proportion of patients who had received previous antibiotic treatment. These results are not surprising considering that prior antibiotic use (particularly cephalosporins and quinolones) is considered one of the major risks for healthcare-associated MRSA infections [34].

Besides these cases of prior antibiotic use, common causative agents such as $S$. pneumoniae and known risk factors for pneumococcal disease, which include many of the comorbidities already mentioned [35], must be taken into account, and the importance of a preventive approach by vaccination for patients with these conditions must be emphasised. In fact, our data also show that, in the S. pneumoniae group, only around one-third of patients had received previous pneumococcal vaccinations despite their baseline risk conditions. This highlights the need for including pneumococcal vaccination 
as part of routine recommendations for the management of these patients. Although serotype information is limited in this study due to the low numbers, it is interesting to note the presence in HCAP of some of the serotypes most frequently found in CAP cases ( 1 or 7F, and in the last few years also 8), and the absence of others usually very common in CAP in adults such as serotype 3 , as observed in previous studies in Spain [36].

In terms of the risk of mortality, neither CURB-65 nor PSI scores were significantly different according to the etiology of pneumonia, in line with the results suggested in the study by Polverino et al [19]. In this Spanish study, no differences were reported between the agents causing pneumonia when HCAP and CAP patients were compared. However, increased mortality was reported in the former group, which led the authors to conclude that the microbiological etiology could not be the principal cause of the difference.

Clinical outcomes in HCAP patients seem to be worse than in CAP patients $[6,19,29]$. As such, they require longer hospital stays [6] and, as our results suggest, this time depends on the microorganism identified as causative agent. Specifically, patients with MRSA pneumonia showed the longest LOS, underlining the problems that this pathology represents in terms of management and costs, as discussed by other authors [37]. Although it was not possible to evaluate the influence of treatment on outcomes, the etiology of pneumonia appears to be an important factor when evaluating the outcomes and treatment required by these patients. On the other hand, patients' status at hospital discharge also differed according to the causative agent and, although death related to pneumonia was not frequent, it was identified as the cause of death in the two patients with MRSA pneumonia who died. This stresses the need for a special focus on resistant pathogens, that, specifically in the case of MRSA, are the cause of increased mortality and morbidity rates $[38,39]$. In line with this, an overall mortality rate of $17.8 \%$ was reported at the end of the study, which is within the range of previous estimates for HCAP patients in Spain $[19,29]$.

From our analysis of cases according to the presence or absence of $S$. pneumoniae or MRSA, our results were particularly relevant in the case of MRSA. First, despite $S$. pneumoniae being the most frequent pathogen reported, we were still able to detect $11.6 \%$ cases positive for MRSA, which not only confirms the results obtained by Polverino et al. [19], but also highlights the growing problem of antibiotic resistance. Our data allows us to envisage a MRSA-positive patient profile in which COPD and previous institutionalisation are frequent underlying factors. Indeed, both have been previously recognised as risk factors for MRSA infection [40-43]. Furthermore, heart failure and chronic respiratory diseases were especially frequent in these patients. This illustrates a common problem in clinical practice in the form of delayed resolution of pneumonia, which is often affected by underlying COPD [44], a comorbidity or underlying factor that we also detected in our population.
The concept of HCAP was firstly introduced to define patients at higher risk of antibiotic-resistant pathogens, who would thus require broad-spectrum therapy $[2,3]$. However, over the course of this study, an intense debate on the usefulness of that definition in identifying these patients has sprung up. Thus, in recent years, several studies have concluded that the definition of HCAP may be sensitive but lacking in specificity and, as a consequence, it does not accurately identify resistant pathogens [11-14]. Indeed, three recent studies have proposed new methods to define patients with an increased risk of developing pneumonia due to multidrug-resistant bacteria [45-47].

This study has some limitations. Firstly, our sample size was limited, preventing us from making predictions and association analysis. Secondly, microbiological and serotype data on S. pneumoniae were also limited, and the impact of certain serotypes on HCAP could not be evaluated. Nevertheless, this multicentre prospective study is the first to evaluate HCAP patients according to the etiology of their pneumonia, thus helping identify risks and prognostic factors that might help to guide treatment in these patients, with enough statistical power.

Therefore, despite the limitations, the results show that the etiology of patients with HCAP is still unknown in most cases, although $S$. pneumoniae appears as the most prevalent microorganism. In patients with COPD, however, a major proportion of HCAP caused by other microorganisms other than S. pneumoniae and MRSA was observed. Importantly, death in the overall population within the first month after discharge was related in most of the cases to pneumonia. Our study offers an updated view of the characteristics and outcomes of these patients in the Spanish population, but further research is needed to provide data to help identifying predictive factors for HCAP to improve prevention and management strategies.

\section{ACKNOWLEDGEMENTS}

Medical writing support was provided by Dr. Almudena Fuster-Matanzo of Medical Statistics Consulting S.L. (Valencia).

\section{MEMBERS OF SOCRATES STUDY GROUP}

Hospital Universitario Marqués de Valdecilla: C. Armiñanzas, C. Gonzalez Rico, J. Calvo. Hospital Universitario A Coruña: L. Castelo. E. Míguez, M. Rodríguez, L. Ramos, J.M. Bravo-Ferrer, E. Sanchez Vidal; Hospital Universitario de Bellvitge: M. Cubero, J. Liñares, A. Simonetti; Hospital Universitario Cruces: B. Vilar; Hospital Universitario Virgen de las Nieves: C. García, S. De Jesús; Hospital General Universitario Gregorio Marañón: A. Burillo, E. Cercenado, E. Gargallo, E. Garcia Leoni; Hospital Universitario San Pedro: L. Garcia, J.M. Azcona

\section{FUNDING}

This study was sponsored by Pfizer. 


\section{COMPETING INTERESTS}

C. Méndez, C. Balseiro and N. Lwoff are employees of Pfizer S.L.U. Madrid, Spain. The rest of the authors have no conflicts of interest.

\section{REFERENCES}

1. Dunn L. Pneumonia: classification, diagnosis and nursing management. Nurs Stand 2005; 19(42):50-4. doi: 10.7748/ ns2005.06.19.42.50.c3901.

2. Kollef MH, Shorr A, Tabak YP, Gupta V, Liu LZ, Johannes RS. Epidemiology and outcomes of health-care-associated pneumonia: results from a large US database of culture-positive pneumonia. Chest 2005; 128(6):3854-62. doi: 10.1378/chest.128.6.3854.

3. American Thoracic Society; Infectious Diseases Society of America. Guidelines for the management of adults with hospital-acquired, ventilator-associated, and healthcare-associated pneumonia. Am J Respir Crit Care Med 2005; 171(4):388-416. doi: 10.1164/rccm.200405-644ST.

4. Shindo Y, Sato S, Maruyama E, Ohashi T, Ogawa M, Hashimoto N et al. Health-care-associated pneumonia among hospitalized patients in a Japanese community hospital. Chest 2009; 135(3):633-640. doi: 10.1378/chest.08-1357.

5. Micek ST, Kollef KE, Reichley RM, Roubinian N, Kollef MH. Health care-associated pneumonia and community-acquired pneumonia: a single-center experience. Antimicrob Agents Chemother 2007; 51(10):3568-73. doi: 10.1128/aac.00851-07.

6. Carratala J, Mykietiuk A, Fernandez-Sabe N, Suarez C, Dorca J, Verdaguer $\mathrm{R}$ et al. Health care-associated pneumonia requiring hospital admission: epidemiology, antibiotic therapy, and clinical outcomes. Arch Intern Med 2007; 167(13):1393-9. doi: 10.1001/ archinte.167.13.1393.

7. Venditti M, Falcone $M$, Corrao $S$, Licata $G$, Serra P, Study Group of the Italian Society of Internal M. Outcomes of patients hospitalized with community-acquired, health care-associated, and hospital-acquired pneumonia. Ann Intern Med 2009; 150(1):19-26. doi: 10.7326/0003-4819-150-1-200901060-00005.

8. Mandell LA, Wunderink RG, Anzueto A, Bartlett JG, Campbell GD, Dean NC et al. Infectious Diseases Society of America/American Thoracic Society consensus guidelines on the management of community-acquired pneumonia in adults. Clin Infect Dis 2007; 44 (Suppl. 2):S27-72. doi: 10.1086/511159.

9. Kollef MH, Napolitano LM, Solomkin JS, Wunderink RG, Bae IG, Fowler VG et al. Health care-associated infection (HAl): a critical appraisal of the emerging threat-proceedings of the HAI Summit. Clin Infect Dis 2008; 47 (Suppl. 2):S55-99; quiz S100-1. doi: 10.1086/590937.

10. Kollef MH. Health care-associated pneumonia: perception versus reality. Clin Infect Dis 2009; 49(12):1875-7. doi: 10.1086/648430.

11. Chalmers JD, Rother C, Salih W, Ewig S. Healthcare-associated pneumonia does not accurately identify potentially resistant pathogens: a systematic review and meta-analysis. Clin Infect Dis 2014;
58(3):330-9. doi: 10.1093/cid/cit734.

12. Falcone M, Russo A, Giannella M, Cangemi R, Scarpellini MG, Bertazzoni $G$ et al. Individualizing risk of multidrug-resistant pathogens in community-onset pneumonia. PLoS One 2015; 10(4):e0119528. doi: 10.1371/journal.pone.0119528.

13. Scholte JB, van Mook WN, Linssen CF. Surveillance cultures in healthcare-associated pneumonia: sense or nonsense? Curr Opin Pulm Med 2014; 20(3):259-71. doi: 10.1097/mcp.0000000000000044.

14. Shorr AF, Zilberberg MD. Role for risk-scoring tools in identifying resistant pathogens in pneumonia: reassessing the value of healthcare-associated pneumonia as a concept. Curr Opin Pulm Med 2015; 21(3):232-8. doi: 10.1097/mcp.0000000000000159.

15. Roson B, Carratala J, Dorca J, Casanova A, Manresa F, Gudiol F. Etiology, reasons for hospitalization, risk classes, and outcomes of community-acquired pneumonia in patients hospitalized on the basis of conventional admission criteria. Clin Infect Dis 2001; 33(2):158-65. doi: 10.1086/321808.

16. Woodhead M. Community-acquired pneumonia in Europe: causative pathogens and resistance patterns. Eur Respir J Suppl 2002; 36:20s-27s. doi: 10.1183/09031936.02.00702002.

17. Almirall J, Bolibar I, Vidal J, Sauca G, Coll P, Niklasson B et al. Epidemiology of community-acquired pneumonia in adults: a population-based study. Eur Respir J 2000; 15(4):757-63. doi: 10.1034/j.1399-3003.2000.15d21.x.

18. Bartlett JG, Mundy LM. Community-acquired pneumonia. N Engl J Med 1995; 333(24):1618-24. doi: 10.1056/nejm 199512143332408.

19. Polverino E, Torres A, Menendez R, Cilloniz C, Valles JM, Capelastegui $A$ et al. Microbial aetiology of healthcare associated pneumonia in Spain: a prospective, multicentre, case-control study. Thorax 2013; 68(11):1007-14. doi: 10.1136/thoraxjnl-2013-203828.

20. Giannella M, Pinilla B, Capdevila JA, Martinez Alarcon J, Munoz $P$, Lopez Alvarez J et al. Pneumonia treated in the internal medicine department: focus on healthcare-associated pneumonia. Clin Microbiol Infect 2012; 18(8):786-94. doi: 10.1111/j.14690691.2011.03757.x.

21. Gollomp K, Rankin SC, White C, Mattei P, Harris MC, Kilpatrick LE et al. Broad-range bacterial polymerase chain reaction in the microbiologic diagnosis of complicated pneumonia. J Hosp Med 2012; 7(1):8-13. doi: 10.1002/jhm.911.

22. Clinical and Laboratory Standards Institute (CLSI). Performance Standards for Antimicrobial Susceptibility Testing: Twentieth Informational Suplement. M100-S20. Clinical and Laboratory Standards, 2010.

23. Carvalho Mda G, Tondella ML, McCaustland K, Weidlich L, McGee L, Mayer LW et al. Evaluation and improvement of real-time PCR assays targeting lytA, ply, and psaA genes for detection of pneumococcal DNA. J Clin Microbiol 2007; 45(8):2460-6. doi: 10.1128/ jcm.02498-06.

24. Charlson ME, Pompei $P$, Ales KL, MacKenzie CR. A new method of classifying prognostic comorbidity in longitudinal studies: development and validation. J Chronic Dis 1987; 40(5):373-83. doi: 10.1016/0021-9681(87)90171-8.

25. Lim WS, van der Eerden MM, Laing R, Boersma WG, Karalus N, Town 
$\mathrm{Gl}$ et al. Defining community acquired pneumonia severity on presentation to hospital: an international derivation and validation study. Thorax 2003; 58(5):377-82. doi: 10.1136/thorax.58.5.377.

26. Fine MJ, Auble TE, Yealy DM, Hanusa BH, Weissfeld LA, Singer DE et al. A prediction rule to identify low-risk patients with community-acquired pneumonia. N Engl J Med 1997; 336(4):243-50. doi: 10.1056/nejm 199701233360402.

27. Bogaert D, De Groot R, Hermans PW. Streptococcus pneumoniae colonisation: the key to pneumococcal disease. Lancet Infect Dis 2004; 4(3):144-54. doi: 10.1016/s1473-3099(04)00938-7.

28. Stenehjem E, Rimland D. MRSA nasal colonization burden and risk of MRSA infection. Am J Infect Control 2013; 41(5):405-10. doi: 10.1016/j.ajic.2012.07.017.

29. Valles J, Martin-Loeches I, Torres A, Diaz E, Seijas I, Lopez MJ et al. Epidemiology, antibiotic therapy and clinical outcomes of healthcare-associated pneumonia in critically ill patients: a Spanish cohort study. Intensive Care Med 2014; 40(4):572-81. doi: 10.1007| s00134-014-3239-2.

30. Lim WS, Macfarlane JT. A prospective comparison of nursing home acquired pneumonia with community acquired pneumonia. Eur Respir J 2001; 18(2):362-8. doi: 10.1183/09031936.01.00204401.

31. Livorsi D, Eckerle MK. Developing local treatment guidelines for healthcare-associated pneumonia. Clin Infect Dis 2014; 59(4):60910. doi: 10.1093/cid/ciu332.

32. Murdoch DR, O'Brien KL, Scott JA, Karron RA, Bhat N, Driscoll AJ et al. Breathing new life into pneumonia diagnostics. J Clin Microbiol 2009; 47(11):3405-8. doi: 10.1128/jcm.01685-09.

33. Torres A, Dorca J, Zalacain R, Bello S, El-Ebiary M, Molinos L et al. Community-acquired pneumonia in chronic obstructive pulmonary disease: a Spanish multicenter study. Am J Respir Crit Care Med 1996; 154(5):1456-61. doi: 10.1164/ajrccm.154.5.8912764.

34. Raygada JL, Levine DP. Methicillin-Resistant Staphylococcus aureus: A Growing Risk in the Hospital and in the Community. Am Health Drug Benefits 2009; 2(2):86-95. PMID: 25126276.

35. Torres A, Blasi F, Dartois N, Akova M. Which individuals are at increased risk of pneumococcal disease and why? Impact of COPD, asthma, smoking, diabetes, and/or chronic heart disease on community-acquired pneumonia and invasive pneumococcal disease. Thorax 2015; 70(10):984-9. doi: 10.1136/thoraxjnl-2015-206780.

36. Menendez R, Espana PP, Perez-Trallero E, Uranga A, Mendez R, Cilloniz $C$ et al. The burden of PCV13 serotypes in hospitalized pneumococcal pneumonia in Spain using a novel urinary antigen detection test. CAPA study. Vaccine 2017; 35(39):5264-5270. doi: 10.1016/j.vaccine.2017.08.007.

37. Andreassen AES, Jacobsen CM, de Blasio B, White R, Kristiansen IS, Elstrøm P. The impact of methicillin-resistant $S$. aureus on length of stay, readmissions and costs: a register based case-control study of patients hospitalized in Norway. Antimicrobial resistance and infection control 2017; 6:74-74. doi: 10.1186/s13756-017-0232-X.

38. Huang SS, Platt R. Risk of methicillin-resistant Staphylococcus aureus infection after previous infection or colonization. Clin Infect Dis 2003; 36(3):281-5. doi: 10.1086/345955.

39. Klevens RM, Morrison MA, Nadle J, Petit S, Gershman K, Ray S et al. Invasive methicillin-resistant Staphylococcus aureus infections in the United States. Jama 2007; 298(15):1763-71. doi: 10.1001/ jama.298.15.1763.

40. Bradley SF. Methicillin-resistant Staphylococcus aureus in nursing homes. Epidemiology, prevention and management. Drugs Aging 1997; 10(3):185-98. doi: 10.2165/00002512-199710030-00003.

41. Gorwitz RJ, Kruszon-Moran D, McAllister SK, McQuillan G, McDougal LK, Fosheim GE et al. Changes in the prevalence of nasal colonization with Staphylococcus aureus in the United States, 20012004. J Infect Dis 2008; 197(9):1226-34. doi: 10.1086/533494.

42. Hidron Al, Kourbatova EV, Halvosa JS, Terrell BJ, McDougal LK, Tenover FC et al. Risk factors for colonization with methicillin-resistant Staphylococcus aureus (MRSA) in patients admitted to an urban hospital: emergence of community-associated MRSA nasal carriage. Clin Infect Dis 2005; 41(2):159-66. doi: 10.1086/430910.

43. O'Sullivan NP, Keane CT. Risk factors for colonization with methicillin-resistant Staphylococcus aureus among nursing home residents. J Hosp Infect 2000; 45(3):206-10. doi: 10.1053/jhin.2000.0759.

44. El Solh AA, Aquilina AT, Gunen H, Ramadan F. Radiographic resolution of community-acquired bacterial pneumonia in the elderly. J Am Geriatr Soc 2004; 52(2):224-9. doi: 10.1111/j.15325415.2004.52059.x.

45. Aliberti $S$, Di Pasquale M, Zanaboni AM, Cosentini R, Brambilla AM, Seghezzi $S$ et al. Stratifying risk factors for multidrug-resistant pathogens in hospitalized patients coming from the community with pneumonia. Clin Infect Dis 2012; 54(4):470-8. doi: 10.1093/ cid/cir840.

46. Shindo $Y$, Ito $R$, Kobayashi $D$, Ando M, Ichikawa $M$, Shiraki A et al. Risk factors for drug-resistant pathogens in community-acquired and healthcare-associated pneumonia. Am J Respir Crit Care Med 2013; 188(8):985-95. doi: 10.1164/rccm.201301-00790C.

47. Shorr AF, Zilberberg MD, Reichley R, Kan J, Hoban A, Hoffman J et al. Validation of a clinical score for assessing the risk of resistant pathogens in patients with pneumonia presenting to the emergency department. Clin Infect Dis 2012; 54(2):193-8. doi: 10.1093/cid/ cir813. 